Research articles

\title{
Implementation of college admission rules ${ }^{\star}$
}

\author{
Tarkk Kara ${ }^{1}$ and Tayfun Sönmez ${ }^{2}$ \\ ' Department of Economics, University of Rochester, Rochester, NY 14627, USA \\ 2 Department of Economics, University of Michigan, Ann Arbor, MI 48109, USA
}

Received: August 22, 1994; revised version July 6, 1995

Summary. We consider both Nash and strong Nash implementation of various matching rules for college admissions problems. We show that all such rules are supersolutions of the stable rule. Among these rules the "lower bound" stable rule is implementable in both senses. The "upper bound" Pareto and individually rational rule is strong Nash implementable yet it is not Nash implementable. Two corollaries of interest are the stable rule is the minimal (Nash or strong Nash) implementable solution that is Pareto optimal and individually rational, and the stable rule is the minimal (Nash or strong Nash) implementable extension of any of its subsolutions.

JEL Classification Numbers: C78, D78.

\section{Introduction}

A class of public decision problems that has been extensively analyzed is the class of two-sided matching problems. (For an exposition of game theoretic modelling and analysis of two-sided matching problems see Roth and Sotomayor [20].) Most of the studies on these problems deal with one-to-one matching. Nevertheless in real life applications many-to-one matching is the most typical case, where one side consists of institutions and the other side consists of individuals: Colleges admit many students, first hire many workers, and hospitals employ many interns. On the other hand, students attend one college, workers work for one firm and interns work for one hospital.

A college admissions problem ${ }^{1}$ consists of two finite disjoint sets of agents (which are interpreted as sets of students and colleges), a vector of natural

\footnotetext{
* We wish to thank Professor William Thomson for his efforts in supervision as well as his useful suggestions. We are grateful to the participants in his reading class, workshops at Bilkent University, University of Rochester, and in particular Jeffrey Banks, Stephen Ching, Bhaskar Dutta, Rangarajan Sundaram and an anonymous referee for their helpful comments.

Correspondence to: T. Kara

1 Gale and Shapley [4] introduces the college admissions problems. Gale and Sotomayor [5], Roth $[15,16,17,18]$, Roth and Sotomayor [19] study various aspects of the college admissions problems.
} 
numbers (where each component is the "capacity" of a particular college), and a preference relation for each agent. Each student has a preference relation over the colleges and not attending a college. Each college has a preference relation over groups of students. An allocation in this context is a matching of colleges and students where no college is matched to more students than its capacity. A student blocks a matching if he prefers not attending to any college to its assignment. A college blocks a matching if he prefers a subset of its assignment to its assignment. A matching is individually rational if it is not blocked by a student or college. A college-student pair blocks a matching if they are not matched to each other, yet the student prefers the college to his assignment and the college prefers a subset of its assignment plus the student to its assignment. A matching is stable if it is not blocked by a student, a college or a college-student pair. A matching is in the core if no coalition can improve on it by its own resources. Following Gale and Shapley [4], Roth [15] shows in a constructive way that the set of stable matchings is non-empty for all college admissions problems. Moreover Roth [17] shows that the set of matchings in the core is equal to the set of stable matchings.

A solution is a correspondence which selects a set of allocations for each problem. We refer to solutions as college admissions rules in this context. Some of the college admission rules of interest are: The stable rule which selects the set of stable matchings for each college admissions problem, the core correspondence which selects the set of matchings in the core, the individually rational rule which selects the set of individually rational matchings, the Pareto rule which selects the set of Pareto efficient matchings, the Pareto efficient and individually rational rule which selects the set of Pareto efficient and individually rational matchings, etc. Note that the stable rule is equal to the core correspondence in this context.

In a decision problem, agents usually have private information about their own preferences. In most cases, it may be unreasonable to expect truthful revelation. However there may be solutions which give agents the incentive to be truthful independently of the other agents' behavior; this property is known as strategy-proofness. On the other hand, it may be possible to reach the allocations selected by a solution in spite of the fact that agents behave strategically, by confronting them with an appropriately constructed "game form." This possibility is the motivation for the theory of implementation. A game form consists of sets of possible actions for each agent (their strategy spaces), and an outcome function, a function that selects an allocation for each profile of actions. Each agent can choose from his strategy space so as to influence the resulting allocation in his favor. The objective is to find a game form such that at equilibrium the desired allocations are obtained. The primary equilibrium concept we consider is Nash equilibrium. (For expositions of implementation theory see Maskin [10], Moore [11] and Thomson [27].)

The results concerning strategy-proofness are quite discouraging in the context of one-to-one matching problems. Roth [14] shows that there is no selection from the stable rule that is strategy-proof. Alcalde and Barberà [1] improve on this result and show that there is no rule that is Pareto efficient, 
individually rational, and strategy-proof. The classs of one-to-one matching problems is a subclass of the class of college admissions problems where the capacity of each college is equal to one. Therefore these negative results directly extend to college admissions problems. ${ }^{2}$ Due to these negative results, in this paper we replace strategy-proofness with the less demanding requirement of Nash implementability. We show that any college admissions rule that is Pareto efficient, individually rational, and Nash implementable should select all the stable matchings (and possibly some other matchings). That is, any college admissions rule satisfying Pareto efficiency, individual rationality, and Nash implementability is a supersolution of the stable rule. Among such college admissions rules, the "lower bound" stable rule is Nash implementable. Therefore the stable rule is the minimal college admissions rule that is Pareto efficient, individually rational and Nash implementable. However the "upper bound" Pareto efficient and individually rational rule is not Nash implementable. The proofs of these implementability results cannot be achieved by the classical technique introduced by Maskin [8]. They are obtained by means of a recent technique developed by Danilov [2].

Nash equilibrium is a strictly non-cooperative concept. If cooperation among the players is possible, the players might do so to influence the outcome in their interest. In such cases it may be more appropriate to consider strong Nash equilibrium as the equilibrium concept. Strong Nash equilibrium (due to Aumann) is the cooperative counterpart of Nash equilibrium. Nash equilibrium is defined in term of unilateral deviations. Strong Nash equilibrium considers deviations by all coalitions. We also consider implementation in strong Nash equilibrium. We show that any college admissions rule that is Pareto efficient, individually rational, and implementable in strong Nash equilibrium is a supersolution of the stable rule. Among such rules both the "lower bound" stable rule and the "upper bound" Pareto efficient and individually rational rule are implementable in strong Nash equilibria.

\section{Definitions and notation}

In this section we first define implementability and related concepts in the framework of general social choice theory. We will make use of these concepts in the framework of college admissions problems.

The set of alternatives is $A$. The set of agents is $N=\{1,2, \ldots, n\}$. For each agent $i \in N, \mathscr{R}_{i}$ is the set of his possible preference relations. The lower contour set of $\boldsymbol{R}_{i}$ at $a \in A$ is $L\left(a, R_{i}\right)=\left\{b \in A: a R_{i} b\right\}$.

\footnotetext{
${ }^{2}$ Sönmez [24] studies strategy-proofness on college admissions problems. He shows that when each college can admit as many students as it wants the stable rule is single-valued and also strategy-proof. Moreover it is the only rule that is Pareto efficient, individually rational and strategy-proof. Finally, he shows that a rule satisfying these properties exists if and only if each college can admit as many students as it wants. Sönmez [25] further studies strategy-proofness on a general class of matching problems and shows that there exists a rule that is Pareto efficient, individually rational and strategy-proof only if the core correspondence is single-valued. Furthermore if there is such a rule it is the core correspondence.
} 
Let $\mathscr{R}=\prod_{i \in N} \mathscr{R}_{i}$. A social choice rule (or simply a rule) is a correspondence $\varphi: \mathscr{R} \rightarrow A$. A preference profile $\widetilde{R}$ is obtained by a monotonic transformation of $\boldsymbol{R}$ at $\boldsymbol{a} \in \boldsymbol{A}$, if $L\left(a, R_{i}\right) \subseteq L\left(a, \widetilde{R}_{i}\right)$ for all $i \in N$. Let $M T(R, a)$ denote the set of preference profiles that are obtained by a monotonic transformation of $R$ at $a$. A rule, $\varphi$, is monotonic if for all $R, \widetilde{R} \in \mathscr{R}$, and for all $a \in \varphi(R)$, if $\widetilde{R} \in M T(R, a)$ then $a \in \varphi(\tilde{R})$. The rule satisfies no veto power if, for all $i \in N$, and for all $R \in \mathscr{R}$, if $A=L\left(a, R_{j}\right)$ for all $j \in N \backslash\{i\}$, then $a \in \varphi(R)$.

A game form is a pair $\Gamma=(X, h)=\left(\prod_{i \in N} X_{i}, h\right)$, where $X_{i}$ is agent $i$ 's strategy space, and $h: X \rightarrow A$ is an outcome function. The pair $(\Gamma, R)$ defines a game. A strategy profile $x \in X$ is a Nash equilibrium for the game $(\Gamma, R)=((X, h), R)$ if and only if for all $i \in N$ and for all $x_{i}^{\prime} \in X_{i}, h(x) R_{i} h\left(x_{i}^{\prime}, x_{-i}\right)$. Let $N(\Gamma, R)$ denote the set of Nash equilibria for the game $(\Gamma, R)$. A strategy profile is a Nash equilibrium if no agent can benefit by a unilateral deviation. The game form $\Gamma$ implements $\varphi$ in Nash equilibria, if $h(N(\Gamma, R))=\varphi(R)$ for all $R \in \mathscr{R}$.

Maskin [8] shows that monotonicity is a necessary condition for Nash implementability. Maskin [8], Williams [28] and Saijo [21] further show that monotonicity and no veto power together are sufficient for Nash implementability.

A college admissions problem is a four-tuple $(C, S, q, R)$. The first two components of the four-tuple $C=\left\{c_{1}, \ldots, c_{n}\right\}$, and; $S=\left\{s_{1}, \ldots, s_{m}\right\}$ are two non-empty, finite and disjoint sets of colleges and students. The third component $q=\left(q_{c_{1}}, \ldots, q_{c_{n}}\right)$, is a vector of positive natural numbers, where $q_{c_{i}}$ is the capacity of college $c_{i} \in C$. The last component $R=\left(R_{i}\right)_{i \in C \cup S}$ is a list of preferences of colleges and students. Let $P_{i}$ denote the strict relation and $I_{l}$ denote the indifference relations associated with the preference relation $R_{i}$ for all $i \in C \cup S$. We consider the case where $C, S$ and $q$ are fixed and hence each preference profile defines a college admissions problem.

The preference relation $R_{s}$ of student $s \in S$ is a linear order on $\Sigma_{s}=\left\{\left\{c_{1}\right\}, \ldots,\left\{c_{n}\right\}, \varnothing\right\}$. Let $\mathscr{R}_{s}$ be the class of all such preference relations for student $s \in S$. The preference relation $R_{c}$ of college $c \in C$ is a linear order on $\Sigma_{c}^{q}=\{G\}_{G \subseteq S}$ and it is responsive (Roth [16]). That is, for all $G \subseteq S$ such that $|G|<q_{c}$,

1. For all $s, s^{\prime} \in S \backslash G, G \cup\{s\} P_{c} G \cup\left\{s^{\prime}\right\}^{\prime} \Leftrightarrow s P_{c} s^{\prime} ;{ }^{3}$

2. For all $s \in S \backslash G, G \cup\{s\} P_{c} G \Leftrightarrow\{s\} P_{c} \varnothing$.

Let $\mathscr{R}_{c}$ be the class of all such preferences for college $c \in C$. Let $\mathscr{R}=\mathscr{R}_{c}^{n} \times \mathscr{R}_{s}^{m}$.

The choice of a college $c$ from a group of students $G \subseteq S$ under the preference $R_{c}$ is defined as

$$
C h_{c}\left(R_{c}, G\right)=\left\{G^{\prime} \subseteq G:\left|G^{\prime}\right| \leq q_{c}, G^{\prime} R_{c} G^{\prime \prime} \text { for all } G^{\prime \prime} \subseteq G \text { such that }\left|G^{\prime \prime}\right| \leq q_{c}\right\}
$$

A matching $\mu$ is a mapping from the set $C \cup S$ into $2^{C \cup S}$ (the class of subsets of $C \cup S$ ) such that:

${ }^{3}$ We write $s$ istead of $\{s\}$. 
1. For all $s \in S,|\mu(s)| \leq 1$ and $\mu(s)=\varnothing$ if $\mu(s) \subseteq C$;

2. For all $c \in C,|\mu(c)| \leq q_{c}$ and $\mu(c)=\varnothing$ if $\mu(c) \subseteq S$;

3. For all $(c, s) \in C \times S, \mu(s)=c$ if and only if $s \in \mu(c){ }^{4}$

For any $i \in C \cup S$, we refer to $\mu(i)$ as the assignment of $i$ at $\mu$. We denote the set of all matchings by $\mathscr{M}$.

In the present context of college admissions problems, $N=C \cup S$ and $A$ is the set of matchings $\mathscr{M}$. Given a preference relation $R_{c}$ of a college $c \in C$, initially defined over $\Sigma_{c}^{q}$, we extend it to the set of matchings $\mathscr{M}$, in the following natural way: $c$ prefers the matching $\mu$ to the matching $\mu^{\prime}$ if and only if it prefers its assignment under $\mu$ to its assignment under $\mu^{\prime}$. We slightly abuse the notation and also use $R_{c}$ to denote this extension. The same can be done for each student $s \in S$.

A college $c \in C$ is acceptable to a student $s \in S$ under a preference profile $R$ if $c P_{s} \varnothing$. A student $s \in S$ is acceptable to a college $c \in C$ under a preference profile $\boldsymbol{R}$ if $s P_{c} \varnothing$. A matching $\mu$ is blocked by a student $s \in S$ under a preference profile $\boldsymbol{R}$ if $\mu(s)$ is not acceptable to $s$ under $R$. A matching $\mu$ is blocked by a college $c$ under a preference profile $\boldsymbol{R}$ if $\mu(c) \neq C h_{c}\left(R_{c}, \mu(c)\right)$. Note that this statement is equivalent to the following under responsive preferences: A matching $\mu$ is blocked by a college $c \in C$ under a preference profile $R$ if there is a student $s \in \mu(c)$ which is not acceptable to $c$ under $R$. A matching $\mu$ is individually rational under a preference profile $R$ if it is not blocked by a student or a college under $R$. We denote the set of all individually rational matchings under $R$ by $\mathscr{I}(R)$.

A matching $\mu$ is blocked by a college-student pair $(c, s) \in C \times S$ under a preference profile $\boldsymbol{R}$ if $c P_{s} \mu(s)$ and $\mu(c) \neq C h_{c}\left(R_{c}, \mu(c) \cup\{s\}\right)$. Note that an individually rational matching $\mu \in \mathscr{I}(R)$ is blocked by a college-student pair $(c, s) \in C \times S$ under a preference profile $R$ if $c P_{s} \mu(s)$ and

1. $s P_{c} \varnothing$ if $|\mu(c)|<q_{c}$,

2. there exists a student $s^{\prime} \in \mu(c)$ with $s P_{c} s^{\prime}$ if $|\mu(c)|=q_{c}$.

A matching $\mu$ is stable under a preference profile $\boldsymbol{R}$ if it is not blocked by a student, a college, or a college-student pair. We denote the set of all stable matchings under $R$ by $\mathscr{P}(R)$. Roth [15] shows that for any $R \in \mathscr{R}$ there exists a matching $\mu_{C}(R) \in \mathscr{P}(R)$ such that

$$
\forall \mu \in \mathscr{S}(R), \forall c \in C \quad \mu_{C}(R)(c) R_{c} \mu(c)
$$

We call the matching $\mu_{C}(R)$ the college-optimal stable matching for the preference profile $R$. There is an analogous matching which favors the students. We refer to this matching as the student-optimal stable matching for the preference profile $R$, and denoted it by $\mu_{S}(R)$.

A matching $\mu$ is Pareto optimal under $R$ if there is no other matching $\mu^{\prime}$ such that $\mu^{\prime}(i) R_{i} \mu(i)$ for all $i \in C \cup S$ and $\mu^{\prime}(j) P_{j} \mu(j)$ for some $j \in C \cup S$. A matching $\mu$ is weakly Pareto optimal under $\boldsymbol{R}$ if there is no other matching $\mu^{\prime}$ such that

${ }^{4}$ We write $c$ instead of $\{c\}$. 
$\mu^{\prime}(i) P_{i} \mu(i)$ for all $i \in C \cup S$. We denote the set of all Pareto optimal matchings under $R$ by $\mathscr{P}(R)$ and the set of all weakly Pareto optimal matchings under $R$ by $\mathscr{P}^{w}(R)$.

In the context of college admissions problems, rules will be referred to as college admission rules. An example of such a rule is the rule that selects the college-optimal stable matching, $\mu_{C}(R)$, for each preference profile $R \in \mathscr{R}$. We call this rule the college-optimal stable rule and denote it by $\mu_{c}$. We define the student-optimal stable rule analogously and denote it by $\mu_{\mathrm{S}}$. Other examples of college admission rules are the one which selects the set of the stable matchings $\mathscr{S}(R)$, the Pareto rule which selects the set of Pareto optimal matchings $\mathscr{P}(R)$, the individually rational rule which selects the set of individually rational matchings $\mathscr{I}(R)$, and the Pareto and individually rational rule which selects the set of Pareto optimal and individually rational matchings $\mathscr{P}(R) \cap \mathscr{I}(R)$ for each preference profile $R \in \mathscr{R}$. We denote the stable rule by $\mathscr{I}$, the Pareto rule by $\mathscr{P}$, the individually rational rule by $\mathscr{I}$, and the Pareto and individually rational rule by $\mathscr{P} \mathscr{F}$.

\section{Monotonic college admission rules}

The stable rule is monotonic. A next natural question is whether there are other subsolutions of the Pareto and individually rational rule that are monotonic. Theorem 1 concerns monotonic subsolutions of the Pareto and individually rational rule. It states and proves that all monotonic solutions that are Pareto efficient and individually rational are supersolutions ${ }^{5}$ of the stable rule. Therefore, if there is any hope of implementing a Pareto efficient and individually rational rule in Nash equilibria, it is the stable rule and its supersolutions.

Theorem 1 Let $\varphi \subseteq \mathscr{P} \mathscr{I}$ be monotonic. Then $\mathscr{S} \subseteq \varphi$.

Proof: Let $\varphi \subseteq \mathscr{P} \mathscr{I}$ be monotonic. Let $R \in \mathscr{R}$ and $\mu \in \mathscr{P}(R)$. We need to show that $\mu \in \varphi(R)$. Let $c \in C$. Define $\underline{\mu}(c)$ as

$$
\underline{\mu}(c)= \begin{cases}\left\{s \in \mu(c): s^{\prime} R_{c} s \text { for all } s^{\prime} \in \mu(c)\right\} & \text { if }|\mu(c)|=q_{c} \\ \varnothing & \text { if }|\mu(c)|<q_{c}\end{cases}
$$

Define the set $\hat{S}(\mu, c)$ as

$$
\hat{S}(\mu, c)=\left\{s \in S: \underline{\mu}(c) P_{c} s P_{c} \varnothing\right\}
$$

Order the elements of $\hat{S}(\mu, c)=\left\{\hat{s}_{1}, \ldots, \hat{s}_{l}\right\}$ such that $\hat{s}_{l} P_{c} \hat{s}_{l-1} \cdots \hat{s}_{2} P_{c} \hat{s}_{1}$. Define the set $Y_{k}(\mu, c)$ as

$$
Y_{k}(\mu, c)=\left\{G \in \Sigma_{c}^{q}: \hat{s}_{k} \notin G,|G|<q_{c}\right\} \quad k=1,2, \ldots, l
$$

Let $R_{c}^{0}=R_{c}$. Given $R_{c}^{k-1}$, let $R_{c}^{k}(k=1, \ldots, l)$ be such that

1. For all $G, G^{\prime} \in \Sigma_{c}^{q} \backslash Y_{k}(\mu, c)$

$$
G P_{c}^{k} G^{\prime} \Leftrightarrow G P_{c}^{k-1} G^{\prime}
$$

${ }^{5}$ A rule $\psi$ is a supersolution of the rule $\varphi$ if $\varphi \subseteq \psi$. 


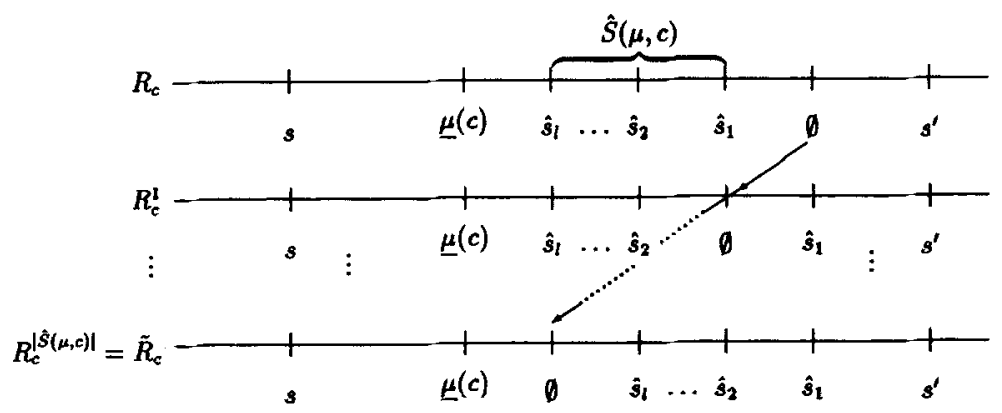

Figure 1.

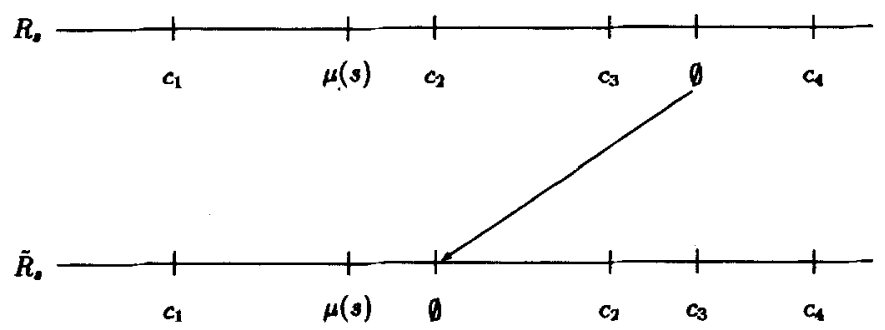

Figure 2.

2. For all $G \in Y_{k}(\mu, c)$

$$
G P_{c}^{k} G \cup\left\{\hat{s}_{k}\right\} \text { and } \nexists \exists G^{\prime} \subseteq S \text { such that } G P_{c}^{k} G^{\prime} P_{c}^{k} G \cup\left\{\hat{s}_{k}\right\}
$$

Note that $R_{c}^{k}$ is responsive for all $k=1, \ldots, l$ and for all $c \in G$ (see Appendix A).

Let $\widetilde{R} \in \mathscr{R}$ be as follows: (see Figure 1 and Figure 2)

1. For all $s \in S$ with $\mu(s)=\varnothing$

$$
\widetilde{R}_{s}=R_{s}
$$

2. For all $s \in S$ wth $\mu(s) \neq \varnothing$

(a) For all $c, c^{\prime} \in C$

$$
c \tilde{P}_{s} c^{\prime} \Leftrightarrow c P_{s} c^{\prime}
$$

(b) $\mu(s) \tilde{P}_{s} \varnothing$ and there does not exists $c \in C \backslash \mu(s)$ such that $\mu(s) \tilde{P}_{s} c \tilde{P}_{s} \varnothing$.

3. For all $c \in C$

$$
\widetilde{R}_{c}=R_{c}^{|\hat{S}(\mu, c)|}
$$

Note that we have $R \in M T(\tilde{R}, \mu)$ (see Appendix B).

We have $\mu \in \mathscr{S}(R) \subseteq \mathscr{I}(R)$, and $\mu \in \mathscr{I}(\tilde{R})$. Suppose there exists a collegestudent pair $(c, s) \in C \times S$ such that $c \widetilde{P}_{s} \mu(s)$. Then we have $c P_{s} \mu(s)$. But $\mu \in \mathscr{P}(R)$, therefore $\underline{\mu}(c) P_{c} s$ and hence $\varnothing \widetilde{P}_{c} s$. Thus no college-student pair blocks $\mu$ under $\widetilde{R}$ and $\mu \in \mathscr{S}(\widetilde{R}) \subseteq \mathscr{P} \mathscr{I}(\widetilde{R})$. 
Let $s \in S$ and $c \in C$ be such that $c \tilde{P}_{s} \mu(s)$. Since $\mu \in \mathscr{P}(\tilde{R})$ we have $\mu(c) \tilde{P}_{c} s$ and hence we have $\varnothing \widetilde{P}_{c} s$. Therefore

$$
\forall \mu^{\prime} \in \mathscr{I}(\widetilde{R}), \quad \forall s \in S \quad \mu(s) \tilde{R}_{s} \mu^{\prime}(s)
$$

Let $c \in C$ and $s \in S \backslash \mu(c)$ be such that $s \tilde{P}_{c} \mu(c)$. Then, since $\mu \in \mathscr{S}(\tilde{R})$ we have $\mu(s) \widetilde{P}_{s} c$ and hence we have $\varnothing \widetilde{P}_{s} c$. This with the individual rationality of $\mu$ under $\tilde{R}$ implies that

$$
\forall \mu^{\prime} \in \mathscr{I}(\tilde{R}), \quad \forall c \in \mathcal{C} \quad \mu(c) \tilde{R}_{c} \mu^{\prime}(c)
$$

and hence $\mathscr{P} \mathscr{I}(\tilde{R})=\{\mu\}$. Therefore $\varphi(\tilde{R})=\{\mu\}$. But $R \in M T(\tilde{R}, \mu)$ and $\varphi$ is monotonic, therefore $\mu \in \varphi(R)$.

Remark 1 This result is a generalization of a result obtained for the one-toone matching problems (marriage problems) in Kara and Sönmez [6].

One of the college admission rules that is often analyzed in the literature is the college-optimal stable rule (student-optimal stable rule). This rule is used to match medical interns and hospitals by the National Resident Matching Program. ${ }^{6}$ One of the corollaries of Theorem 1 is that the college-optimal stable rule (student-optimal stable rule) is not monotonic. Therefore the college-optimal stable rule is not Nash implementable, and hence it is natural to ask how close it is to being Nash implementable. One appealing operation consists of expanding the rule such that the resulting rule is Nash implementable, and therefore monotonic. Among the possible expansions a minimal one is the most desirable. This is the motivation for the following definition due to Sen [22]. Given $\varphi: \mathscr{R} \rightarrow A$, the minimal monotonic extension of $\boldsymbol{\varphi}$, mme $(\varphi)$, is defined by

$$
m m e(\varphi)=\bigcap\{\psi: \mathscr{R} \rightarrow A: \psi \supseteq \varphi, \text { where } \psi \text { is monotonic }\}
$$

Note that $m m e(\varphi)$ is well defined as the feasible rule, the rule that selects the set of all alternatives for each preference profile, is monotonic. We also have $m m e(\varphi)$ monotonic as monotonicity is closed under intersection. (To see some applications of the concept of minimal monotonic extensions in the classical domain see Thomson [26].)

Another corollary to Theorem 1 is that the stable rule is the minimal monotonic extension of any of its subsolutions. In particular, it is the minimal monotonic extension of the college-optimal stable rule (student-optimal stable rule).

Corollary 1 Let $\varphi \subseteq \mathscr{S}$ be monotonic. Then $\varphi=\mathscr{S}$.

Proof: Follows from monotonicity of the stable rule and Theorem 1.

Any matching that is not stable has little chance to survive in real life applications and this observation highlights the importance of this corollary. Consider the hospital-intern matching in the United States. After being matched to a hospital it usually takes an intern only a few telephone calls to

${ }^{6}$ See Roth [15]. 
identify a preferable match. If a hospital can also benefit from this arrangement, there is no reason to expect the intern and the hospital to accept the assignment of the National Resident Matching Program. Note that in the United States the participation in this system is voluntary and the arrangements of the National Resident Matching Program are not legally binding.

\section{Nash implementable college admission rules}

The next natural question is whether there are subsolutions of the Pareto and individually rational rule that are Nash implementable. Due to Theorem 1, we need to concentrate on the supersolutions of the stable rule. A natural point of departure is checking whether the stable rule itself is Nash implementable.

Monotonicity is a necessary condition for Nash implementability. Monotonicity together with no veto power are sufficient conditions for Nash implementability. The stable rule is monotonic. Yet it does not satisfy no veto power. Hence we cannot determine whether the stable rule is Nash implementable by appealing to these results. Nevertheless, the next theorem shows that the stable rule is Nash implementable as long as there are at least three agents. We will need further notation and results to prove the theorem. For this, we will return to the general context.

Let $\varphi: \mathscr{R} \rightarrow A$ and $B \subseteq A$. An alternative $b \in B$ is essential for agent $i \in N$ in $B$ for $\varphi$ if

$$
\exists R \in \mathscr{R} \quad L\left(b, R_{i}\right) \subseteq B \quad \text { and } \quad b \in \varphi(R)
$$

We denote the set of esssential alternatives for agent $i \in N$ in $B$ for $\varphi$ by $E(\varphi, i, B)$. A rule, $\varphi$, is essentially monotonic if for all $R, \widetilde{R} \in \mathscr{R}$ and for all $a \in \varphi(R)$, if $E\left(\varphi, i, L\left(a, R_{i}\right)\right) \subseteq L\left(a, \widetilde{R}_{i}\right)$ for all $i \in N$, then $a \in \varphi(\tilde{R})$.

Danilov [2] shows that if $|N| \geq 3$ then $\varphi$ is Nash implementable if and only if it is essentially monotonic.

Danilov proves his result on a domain where preferences are linear orders on $A$. Yamato [29] generalizes his result as follows: Let $\mathscr{R}$ be such that, for all $a \in A, R \in \mathscr{R}, i \in N$, and $b \in L\left(a, R_{i}\right)$, there exists $R^{\prime} \in \mathscr{R}$ such that $L\left(b, R_{i}^{\prime}\right)=L\left(a, R_{i}\right)$ and for all $j \neq i, L\left(b, R_{j}^{\prime}\right)=A$. Then, if $|N| \geq 3$, a social choice rule $\varphi$ is Nash implementable if and only if it is esssentially monotonic.

Our domain does not satisfy Danilov's domain assumption. Nevertheless it does satisfy Yamato's. Therefore in the proof of the following theorem we will use Yamato's result.

Lemma 1 For all $R \in \mathscr{R}, \mu \in \mathscr{S}(R), \mathcal{c} \in C, s \in S$ we have

$$
\begin{aligned}
& E\left(\mathscr{P}, c, L\left(\mu, R_{c}\right)\right)=\left\{\mu^{\prime} \in L\left(\mu, R_{c}\right): \forall G \subseteq \mu^{\prime}(c) \quad \mu(c) R_{c} G\right\} \\
& E\left(\mathscr{P}, s, L\left(\mu, R_{s}\right)\right)=L\left(\mu, R_{s}\right)
\end{aligned}
$$

Proof: Let us first consider the colleges. Let $R \in \mathscr{R}, \mu \in \mathscr{S}(R)$ and $c \in C$. Let

$$
\hat{L}\left(\mu, R_{c}\right)=\left\{\mu^{\prime} \in L\left(\mu, R_{c}\right): \forall G \subseteq \mu^{\prime}(c) \quad \mu(c) R_{c} G\right\}
$$


Claim 1: $E\left(\mathscr{S}, c, L\left(\mu, R_{c}\right)\right) \subseteq \widehat{L}\left(\mu, R_{c}\right)$.

Let $\mu^{\prime} \in E\left(\mathscr{S}, c, L\left(\mu, R_{c}\right)\right)$. Then there exists $R^{\prime} \in \mathscr{R}$ such that

$$
\mu^{\prime} \in \mathscr{P}\left(R^{\prime}\right) \text { and } L\left(\mu^{\prime}, R_{c}^{\prime}\right) \subseteq L\left(\mu, R_{c}\right)
$$

Therefore $\mu^{\prime} \in L\left(\mu, R_{c}\right)$. As $\mu^{\prime} \in \mathscr{S}\left(R^{\prime}\right)$ we have

which implies

$$
\forall G \subseteq \mu^{\prime}(c) \quad \mu^{\prime}(c) R_{c}^{\prime} G
$$

There $\mu^{\prime} \in \hat{L}\left(\mu, R_{c}\right)$.

$$
\forall G \subseteq \mu^{\prime}(c) \quad \mu(c) R_{c} G
$$

Claim 2: $\hat{L}\left(\mu, R_{c}\right) \subseteq E\left(\mathscr{S}, c, L\left(\mu, R_{c}\right)\right)$

Let $\mu^{\prime} \in \hat{L}\left(\mu, R_{c}\right)$. Let $R^{\prime} \in \mathscr{R}$ be such that

1. $\forall s \in S, \forall c \in C \backslash \mu^{\prime}(s) \quad \mu^{\prime}(s) R_{s}^{\prime} \varnothing P_{s}^{\prime} c$.

2. $\forall c^{\prime} \in C \backslash\{c\}, \forall G \in \sum_{c^{\prime}}^{q} \quad \mu^{\prime}\left(c^{\prime}\right) R_{c^{\prime}}^{\prime} G$.

3. $\forall G \subseteq \mu(c), \forall G^{\prime} \Phi \mu(c) \quad G^{\prime} P_{c}^{\prime} \mu(c) R_{c}^{\prime} G R_{c}^{\prime} \varnothing$.

(Note that in the preference profile $R^{\prime}$ the colleges' preferences are compatible with responsive preferences.)

By construction we have $\mu^{\prime} \in \mathscr{S}\left(R^{\prime}\right)$ and

$$
L\left(\mu^{\prime}, R_{c}^{\prime}\right)=\left\{\tilde{\mu} \in \mathscr{M}: \exists G \subseteq \mu^{\prime}(c) \tilde{\mu}(c)=G\right\}
$$

As $\mu^{\prime} \in \hat{L}\left(\mu, R_{c}\right)$ we have $\mu(c) R_{c} \tilde{\mu}(c)$ for all $\tilde{\mu} \in L\left(\mu^{\prime}, R_{c}^{\prime}\right)$, i.e., $L\left(\mu^{\prime}, R_{c}^{\prime}\right) \subseteq L\left(\mu, R_{c}\right)$. Therefore $\mu^{\prime} \in E\left(\mathscr{S}, c, L\left(\mu, R_{c}\right)\right)$.

This shows that

$$
E\left(\mathscr{S}, c, L\left(\mu, R_{c}\right)\right)=\hat{L}\left(\mu, R_{c}\right)
$$

By similar arguments it can be shown that

$$
E\left(\mathscr{Y}, s, L\left(\mu, R_{s}\right)\right)=\hat{L}\left(\mu, R_{s}\right)=\left\{\mu^{\prime} \in L\left(\mu, R_{s}\right): \forall G \subseteq \mu^{\prime}(s) \mu(s) R_{s} G\right\}
$$

Each student has a capacity equal to one and $\mu \in \mathscr{P}(R) \subseteq \mathscr{I}(R)$, which implies $\widehat{L}\left(\mu, R_{s}\right)=L\left(\mu, R_{s}\right)$. Therefore

$$
E\left(\mathscr{S}, s, L\left(\mu, R_{s}\right)\right)=L\left(\mu, R_{s}\right)
$$

Note that for all $R \in \mathscr{R}$, for all $c \in C$, and for all $\mu \in \mathscr{I}(R), \mu \in \hat{L}\left(\mu, R_{c}\right)=$ $E\left(\mathscr{S}, c, L\left(\mu, R_{\ell}\right)\right)$.

Theorem 2 Let $|C \cup S| \geq 3$. The stable rule is implementable in Nash equilibria.

Proof: Let $|C \cup S| \geq 3$. Let $R, R^{\prime} \in \mathscr{R}$ and $\mu \in \mathscr{S}(R)$ be such that

$$
\forall i \in C \cup S \quad E\left(\mathscr{S}, i, L\left(\mu, R_{i}\right)\right) \subseteq L\left(\mu, R_{i}^{\prime}\right)
$$

(1) Let $s \in S$. Let $\mu_{I}$ be such that $\mu_{I}\left(s^{\prime}\right)=\varnothing$ for all $s^{\prime} \in S$. We have $\mu \in \mathscr{P}(R) \subseteq \mathscr{F}(R)$ and therefore

$$
\mu_{I} \in L\left(\mu, R_{s}\right)=E\left(\mathscr{S}, s, L\left(\mu, R_{s}\right)\right) \subseteq L\left(\mu, R_{s}^{\prime}\right)
$$

Hence $s$ will not block $\mu$ under $R_{s}^{\prime}$ alone. 
(2) Let $c \in C$. Note that if $\tilde{\mu} \in \hat{L}\left(\mu, R_{c}\right)$ and $\hat{\mu}$ is such that $\hat{\mu}(c)=\tilde{\mu}(c) \backslash\{s\}$ for some $s \in \tilde{\mu}(c)$, then $\hat{\mu} \in \hat{L}\left(\mu, R_{c}\right)$. We have $\mu \in \hat{L}\left(\mu, R_{c}\right)$ therefore for all $\hat{\mu}$ such that $\hat{\mu}(c)=\mu(c) \backslash\{s\}$ for some $s \in \mu(s)$

$$
\hat{\mu} \in \hat{L}\left(\mu, R_{c}\right)=E\left(\mathscr{S}, c, L\left(\mu, R_{c}\right)\right) \subseteq L\left(\mu, R_{c}^{\prime}\right)
$$

Hence $c$ will not block $\mu$ under $R_{c}^{\prime}$ alone.

(3) Let $(c, s) \in C \times S$. Suppose $(c, s)$ blocks $\mu$ under $R^{\prime}$. Then

$$
c P_{s}^{\prime} \mu(s) \text { and } s \in C h_{c}\left(R_{c}^{\prime}, \mu(c) \cup\{s\}\right)
$$

Let $\tilde{\mu} \in \mathscr{M}$ be such that $\tilde{\mu}(c)=C h_{c}\left(R_{c}^{\prime}, \mu(c) \cup\{s\}\right)$. We have $E\left(\mathscr{S}, s, L\left(\mu, R_{s}\right)\right)=$ $L\left(\mu, R_{s}\right)$, therefore $L\left(\mu, R_{s}\right) \subseteq L\left(\mu, R_{s}^{\prime}\right)$ which implies

$$
c P_{s} \mu(s)
$$

Furthermore suppose that $\mu(c) R_{c} \tilde{\mu}(c)$. Let $G=\tilde{\mu}(c) \backslash\{s\}$. Note that $G \subseteq \mu(c)$. We have $\mu \in \mathscr{I}(R)$ therefore $\mu(c) R_{c} G R_{c} G^{\prime}$ for all $G^{\prime} \subseteq G$. This together with the responsiveness of the preferences implies that $G \cup\{s\}=\tilde{\mu}(c) R_{c} G^{\prime} \cup\{s\}$ for all $G^{\prime} \subseteq G$ and therefore $\mu(c) R_{c} G^{\prime \prime}$ for all $G^{\prime \prime} \subseteq \tilde{\mu}(c)$. That is $\tilde{\mu} \in E\left(\mathscr{S}, \mathcal{c}, L\left(\mu, R_{c}\right)\right)$. But $E\left(\mathscr{P}, c, L\left(\mu, R_{c}\right)\right) \subseteq L\left(\mu, R_{c}^{\prime}\right)$ therefore $\mu(c) R_{c}^{\prime} \tilde{\mu}(c)$ which is a contradiction to $\tilde{\mu}(c)=C h_{c}\left(R_{c}^{\prime}, \mu(c) \cup\{s\}\right)$. Therefore

$$
\tilde{\mu}(c) P_{c} \mu(c)
$$

We also have $c P_{s} \mu(s)$, therefore $(c, s)$ blocks $\mu$ under $R$. This is a contradiction to $\mu \in \mathscr{S}(R)$. Hence $(c, s)$ will not block $\mu$ under $R^{\prime}$.

This shows that $\mu \in \mathscr{S}\left(R^{\prime}\right)$, therefore the stable rule is essentially monotonic and hence it is implementable in Nash equilibria.

Note that Theorem 1 and Theorem 2 together imply that the stable rule is the minimal Nash implementable subsolution ${ }^{7}$ of the Pareto and individually rational rule. The next corollary states that (as long as there are three or more agents) the stable rule is the minimal Nash implementable extension of any of its subsolutions.

Corollary 2 Let $|C \cup S| \geq 3$ and $\varphi \subseteq \mathscr{S}$ be Nash implementable. Then $\varphi=\mathscr{P}$. Proof: Follows from Theorem 2 and Corollary 1.

The next step is implementing the stable rule in Nash equilibria. As we have satisfied the necessary and sufficient conditions noted in Danilov [2], the game form introduced in the paper can be used to implement the stable rule in Nash equilibria.

Moore and Repullo [12], Dutta and Sen [3], and Sjöström [23] provide necessary and sufficient conditions for Nash implementability whenever there are two agents. Using their conditions, it is straightforward to show that the stable rule is not Nash implementable whenever $|C|=|S|=1$. See Kara and Sönmez [7] for an independent proof.

\footnotetext{
7 The solution $\psi \subseteq \varphi$ is the minimal Nash implementable subsolution of $\varphi$ if it is Nash implementable and $\psi \subseteq \phi$ for all $\phi \subseteq \varphi$ that is Nash implementable.
} 
What about the Pareto and individually rational rule itself? Before answering this question let us first consider the Pareto rule and the individually rational rule. It is known that the Pareto rule is monotonic when preferences are strict. (See for example Palfrey and Srivastava [13].) It also satisfies no veto power. Therefore the Pareto rule is Nash implementable. ${ }^{8}$ The individually rational rule is also monotonic. Yet it does not satisfy no veto power. Nevertheless the individually rational rule is implementable in Nash equilibria. We propose a simple game form to implement the individually rational rule in Nash equilibria. In this game form each student announces a college and each college announces a group of students.

Let $\Lambda=(X, h)$ be as follows: $X_{c}=\Sigma_{c}^{q}$ for all $c \in C$ and $X_{s}=\Sigma_{s}$ for all $s \in S$; $h: X \rightarrow \mathscr{M}$ is defined as

$$
\forall x \in X, \forall s \in S \quad h(x)(s)= \begin{cases}x_{s} & \text { if } x_{s} \neq \varnothing \text { and } s \in x_{x_{s}} \\ \varnothing & \text { otherwise }\end{cases}
$$

Proposition 1 The game form $A$ implements the individually rational rule in Nash equilibria.

\section{Proof:}

(1) $\mathscr{I}(R) \subseteq h(N(\Lambda, R))$.

Let $\mu \in \mathscr{I}(R)$. Let $x \in X$ be such that $x_{i}=\mu(i)$ for all $i \in C \cup S$. Then $h(x)=\mu$. For all $s \in S$, for all $x_{s}^{\prime} \in X_{s}$,

$$
h\left(x_{s}^{\prime}, x_{-s}\right)(s) \in\{\mu(s), \varnothing\}
$$

and we have $\mu(s) R_{s} \varnothing$ by $\mu \in \mathscr{I}(R)$. Therefore no student gains by a unilateral deviation.

For all $c \in C$, for all $x_{c}^{\prime} \in X_{c}$

$$
h\left(x_{c}^{\prime}, x_{-c}\right)(c) \in\left\{G \in \Sigma_{c}^{q}: G \subseteq \mu(c)\right\}
$$

We have $\mu(c) R_{c} G$ for all $G \in \mu(c)$ by $\mu \in \mathscr{F}(R)$. Therefore no college gains by a unilateral deviation.

Hence $x \in N(\Lambda, R)$ and $\mu=h(x) \in h(\Lambda, R)$.

(2) $h(N(\Lambda, R)) \subseteq \mathscr{I}(R)$.

Let $\mu \in h(N(\Lambda, R))$ and $x \in N(\Lambda, R)$ be such that $h(x)=\mu$.

Let $s \in S, x_{s}^{\prime}=\varnothing$. Then $h\left(x_{s}^{\prime}, x_{-s}\right)(s)=\varnothing$. But $x \in N(\Lambda, R)$, therefore

$$
\mu(s)=h(x)(s) R_{s} h\left(x_{s}^{\prime}, x_{-s}\right)(s)=\varnothing
$$

Let $c \in C, s \in \mu(c), x_{c}^{\prime}=\mu(c) \backslash\{s\}$. Then $h\left(x_{c}^{\prime}, x_{-c}\right)(c)=\mu(c) \backslash\{s\}$. But $x \in N(\Lambda, R)$ therefore

$$
\mu(c)=h(x)(c) R_{c} h\left(x_{c}^{\prime}, x_{-c}\right)(c)=\mu(c) \backslash\{s\}
$$

Hence $\mu \in \mathscr{I}(R)$.

\footnotetext{
${ }^{8}$ Let the weak Pareto rule $\mathscr{P}^{w}$, be the matching rule which selects the set of weak Pareto optimal matchings for each preference profile $R \in \mathscr{R}$. Similar results holds if the weak Pareto rule is replaced by the Pareto rule.
} 
The Pareto and individually rational rule is monotonic since both the Pareto rule and the individually rational rule are monotonic. However the Pareto and individually rational rule does not satisfy no veto power, so that once again Maskin's result does not apply. The next proposition shows that the Pareto and individually rational rule is not implementable in Nash equilibria.

Lemma 2 For all $R \in \mathscr{R}, \mu \in \mathscr{P} \mathscr{I}(R), c \in C, s \in S$ we have

$$
\begin{aligned}
& E\left(\mathscr{P} \mathscr{I}, c, L\left(\mu, R_{c}\right)\right)=\left\{\mu^{\prime} \in L\left(\mu, R_{c}\right): \forall G \subseteq \mu^{\prime}(c) \mu(c) R_{c} G\right\} \\
& E\left(\mathscr{P} \mathscr{I}, s, L\left(\mu, R_{s}\right)\right)=L\left(\mu, R_{s}\right)
\end{aligned}
$$

Proof: Similar to Lemma 1.

Proposition 2 The Pareto and individually rational rule is not implementable in Nash equilibria.

Proof: Let $S=\left\{s_{1}, s_{2}, s_{3}, s_{4}\right\}, C=\left\{c_{1}, c_{2}\right\}, q_{c_{1}}=q_{c_{2}}=2$ and $R, \tilde{R} \in \mathscr{R}$ be such that

$$
\begin{array}{ccccccl}
R_{c_{1}} & \tilde{R}_{c_{1}} & R_{c_{2}}=\tilde{R}_{c_{2}} & R_{s_{1}}=\tilde{R}_{s_{1}} & R_{s_{2}}=\tilde{R}_{s_{2}} & R_{s_{3}}=\tilde{R}_{s_{3}} & R_{s_{4}}=\tilde{R}_{s_{4}} \\
\left\{s_{1}, s_{2}\right\} & \left\{s_{1}, s_{2}\right\} & \left\{s_{1}, s_{2}\right\} & c_{1} & c_{2} & c_{2} & c_{1} \\
\left\{s_{1}, s_{3}\right\} & \left\{s_{1}, s_{3}\right\} & \left\{s_{1}, s_{3}\right\} & c_{2} & c_{1} & c_{1} & c_{2} \\
\left\{s_{1}\right\} & \left\{s_{1}\right\} & \left\{s_{2}, s_{3}\right\} & \varnothing & \varnothing & \varnothing & \varnothing \\
\left\{s_{2}, s_{4}\right\} & \left\{s_{1}, s_{4}\right\} & \left\{s_{1}, s_{4}\right\} & & & & \\
\left\{s_{1}, s_{4}\right\} & \left\{s_{2}, s_{3}\right\} & \left\{s_{1}\right\} & & & & \\
\left\{s_{2}\right\} & \left\{s_{2}\right\} & \left\{s_{2}, s_{4}\right\} & & & & \\
\left\{s_{2}, s_{4}\right\} & \left\{s_{2}, s_{4}\right\} & \left\{s_{2}\right\} & & & & \\
\left\{s_{3}\right\} & \left\{s_{3}\right\} & \left\{s_{3}, s_{4}\right\} & & & & \\
\left\{s_{3}, s_{4}\right\} & \left\{s_{3}, s_{4}\right\} & \left\{s_{3}\right\} & & & & \\
\varnothing & \varnothing & \left\{s_{4}\right\} & & & & \\
\left\{s_{4}\right\} & \left\{s_{4}\right\} & \varnothing & & & &
\end{array}
$$

Let $\mu \in \mathscr{M}$ be such that $\mu\left(c_{1}\right)=\left\{s_{2}, s_{3}\right\}$ and $\mu\left(c_{2}\right\}=\left\{s_{1}, s_{4}\right\}$. Note that $\mu \in \mathscr{P} \mathscr{I}(R)$. We have

and

$$
\begin{aligned}
& E\left(\mathscr{P} \mathscr{I}, c_{1}, L\left(\mu, R_{c_{1}}\right)\right)=L\left(\mu, R_{c_{1}}\right) \backslash\left\{s_{1}, s_{4}\right\} \\
& E\left(\mathscr{P} \mathscr{I}, c_{2}, L\left(\mu, R_{c_{2}}\right)\right)=L\left(\mu, R_{c_{2}}\right)
\end{aligned}
$$

We also have

$$
\forall s \in S \quad E\left(\mathscr{P} \mathscr{I}, s, L\left(\mu, R_{s}\right)\right)=L\left(\mu, R_{s}\right)
$$

$$
\forall i \in C \cup S E\left(\mathscr{P} \mathscr{I}, i, L\left(\mu, R_{i}\right)\right) \subseteq L\left(\mu, \tilde{R}_{i}\right)
$$

Let $\mu^{\prime} \in \mathscr{M}$ be such that $\mu^{\prime}\left(c_{1}\right)=\left\{s_{1}, s_{4}\right\}$ and $\mu^{\prime}\left(c_{2}\right)=\left\{s_{2}, s_{3}\right\}$. Note that $\mu^{\prime}(i) \tilde{P}_{i} \mu(i)$ for all $i \in C \cup S$. Therefore $\mu \notin \mathscr{P}^{w}(\widetilde{R})$ and $\mu \notin \mathscr{P} \mathscr{I}(\tilde{R})$ which implies that $\mathscr{P} \mathscr{I}$ is not essentially monotonic and hence not Nash implementable.

Remark 2 Even though both the Pareto rule and the individually rational rule are Nash implementable, the Pareto and individually rational rule is not. This illustrates that the Nash implementability (unlike monotonicity) is not closed under intersection. 
Table 1

\begin{tabular}{lcccl}
\hline & $\begin{array}{c}\text { No veto } \\
\text { power }\end{array}$ & Monotonicity & $\begin{array}{c}\text { Nash } \\
\text { implementable }\end{array}$ & Notes \\
\hline $\mathscr{S}$ & No & Yes & Yes & $\begin{array}{l}\text { We use Danilov's results to show } \\
\text { that } \mathscr{P} \text { is Nash implementable }\end{array}$ \\
\hline$\mu_{S}$ & No & No & No & Follows from Maskin's result \\
\hline$\varphi \subseteq \mathscr{P}$ & No & No & No & Follows from Maskin's result \\
\hline $\mathscr{P}$ & Yes & Yes & Yes & Follows from Maskin's result \\
\hline $\mathscr{P}^{w}$ & Yes & Yes & Yes & Follows from Maskin's result \\
\hline $\mathscr{I}$ & No & Yes & Yes & $\begin{array}{l}\text { We provide a simple game form } \\
\text { to implement } \mathscr{I} \text { in Nash equilibria }\end{array}$ \\
\hline $\mathscr{P} \mathscr{I}$ & No & Yes & No & $\begin{array}{l}\text { We use Danilov's result to show } \\
\text { that } \mathscr{P} \mathscr{I} \text { is not Nash implementable }\end{array}$ \\
\hline $\mathscr{P}^{w} \mathscr{I}$ & No & Yes & No & $\begin{array}{l}\text { We use Danilov's result to show } \\
\text { that } \mathscr{P} \mathscr{P}^{\mathscr{I}} \text { is not Nash } \\
\text { implementable }\end{array}$ \\
\hline
\end{tabular}

Remark 3 Let the weak Pareto and individually rational rule be the matching rule which selects the set of weakly Pareto optimal and individually rational matchings $\mathscr{P}^{w}(R) \cap \mathscr{I}(R)$ for each preference profile $R \in \mathscr{R}$. We denote the weak Pareto and individually rational rule by $\mathscr{P}^{w} \mathscr{I}$. The proof of Proposition 2 can be modified to show that the weak Pareto and individually rational rule is not implementable in Nash equilibria.

Remark 4 Kara and Sönmez [6] shows that the Pareto and individually rational rule is Nash implementable in the class of one-to-one matching problems.

We summarize these results in Table 1:

\section{Strong Nash implementable college admission rules}

So far we have considered Nash equilibrium as the equilibrium concept. Nash equilibrium is a strictly non-cooperative concept. If cooperation among the players is possible, the players might cooperate to influence the outcome towards their interest. In such cases it might be more appropriate to consider strong Nash equilibrium as the equilibrium concept. Strong Nash equilibrium (due to Aumann) is the cooperative counterpart of the Nash equilibrium. Nash equilibrium is defined in terms of unilateral deviations. Strong Nash equilibrium considers deviations by all coalitions. In this section we consider strong Nash equilibrium as the equilibrium concept.

A strategy profile $x \in X$ is a strong Nash equilibrium for the game $(\Gamma, R)=((X, h), R)$ if and only if for all $G \subseteq N$, and for all $x_{G}^{\prime} \in X_{G}$, either 
(i) there is an agent $i \in G$ such that $h(x) P_{i} h\left(x_{G}^{\prime}, x_{-G}\right)$, or

(ii) for any agent $i \in G, h(x) R_{i} h\left(x_{G}^{\prime}, x_{-G}\right)$.

Let $S N(\Gamma, R)$ denote the set of strong Nash equilibria for the game $(\Gamma, R)$.

A strategy profile is a strong Nash equilibrium if no coalition of agents can weakly benefit all and strictly benefit at least one of its agents by a collective deviation. The game form $\Gamma$ implements $\varphi$ in strong Nash equilibria, if $h(S N(\Gamma, R))=\varphi(R)$ for all $R \in \mathscr{R}$.

Monotonicity is not necessary for strong Nash implementation. ${ }^{9}$ Nevertheless whenever the preferences are strict, monotonicity is necessary for strong Nash implementation. Therefore Theorem 1 implies that if we have any hope of implementing a Pareto efficient and individually rational matching rule in strong Nash equilibria, it is the stable rule and its supersolutions. The next proposition shows that the stable rule is implementable in strong Nash equilibria. We will need further definitions and results to prove the proposition.

We define the set of stable matchings to be the set of matchings which are not blocked by coalitions of size one or two. It is not obvious that this notion is adequate as we might need to consider coalitions of bigger size. This is the motivation for the following definitions.

A matching $\mu \in \mathscr{M}$ is blocked by a coalition $G \subseteq C \cup S$ if there exists another matching $\mu^{\prime} \in \mathscr{M}$ such that for all students $s \in G \cap S$ and for all colleges $c \in G \cap C$

1. $\mu^{\prime}(s) \in G$ (i.e., every student in $G$ who is matched by $\mu^{\prime}$ is matched to a college in $G)$;

2. $\mu^{\prime}(s) P_{s} \mu(s)$ (i.e., every student in $G$ prefers his or her new matching to his or her old one);

3. $i \in \mu^{\prime}(c) \Rightarrow i \in G \cup \mu(c)$ (i.e., every college in $G$ is matched at $\mu^{\prime}$ to new students from $G$, although it may continue to be matched with some of its "old" students from $\mu(c))$;

4. $\mu^{\prime}(c) P_{c} \mu(c)$ (i.e., every college in $G$ prefers its new set of students to its old ones)

A matching $\mu$ is group stable under $\boldsymbol{R}$ if it is not blocked by any coalition.

Roth [16] shows that as long as the preferences for the colleges are responsive, group stability is equivalent to stability for college admissions problems. That is, a matching is group stable if and only if it is stable.

Recall that we defined the game form $A$ as follows: $X_{c}=\Sigma_{c}^{q}$ for all $c \in C$ and $X_{s}=\Sigma_{s}$ for all $s \in S, h: X \rightarrow \mathscr{M}$ is such that

$$
\forall x \in X, \forall s \in S \quad h(x)(s)= \begin{cases}x_{s} & \text { if } x_{s} \neq \varnothing \text { and } s \in x_{x_{s}} \\ \varnothing & \text { otherwise }\end{cases}
$$

\footnotetext{
${ }^{9}$ Maskin [9] considers implementation in the following weaker notion of strong Nash equilibria: A strategy should be such that no coalition of agents can benefit all of its members by a collective deviation. He shows that monotonicity is necessary for implementation in this notion of equilibrium.
} 
In the last section we show that the game form $\Lambda$ implements the individually rational rule in Nash equilibria. The next proposition shows that the same game form implements the stable rule in strong Nash equilibria.

Proposition 3 The game form $\Lambda$ implements the stable rule in strong Nash equilibria.

Proof:

Claim 1: $h(S N(\Lambda, R)) \subseteq \mathscr{P}(R)$.

Let $\mu \in h(S N(\Lambda, R))$ and $x \in S N(\Lambda, R)$ be such that $h(x)=\mu$.

Let $s \in S$, and $x_{s}^{\prime}=\varnothing$. Then $h\left(x_{s}^{\prime}, x_{-s}\right)(s)=\varnothing$. But $x \in S N(\Lambda, R) \subseteq N(\Lambda, R)$, therefore

$$
\mu(s)=h(x)(s) R_{s} h\left(x_{s^{\prime}}^{\prime}, x_{-s}\right)(s)=\varnothing
$$

Let $c \in C, G \subseteq \mu(c)$, and $x_{c}^{\prime}=G$. Then $h\left(x_{c}^{\prime}, x_{-c}\right)(c)=G$. But $x \in S N(\Lambda, R) \subseteq$ $N(\Lambda, R)$, therefore

$$
\mu(c)=h(x)(c) R_{c} h\left(x_{c}^{\prime}, x_{-c}\right)(c)=G
$$

Thus, no student or college will block $\mu$ under $R$ individually and hence $\mu \in \mathscr{T}(R)$.

Finally let $(c, s) \in C \times S$ be such that $s \notin \mu(c)$. We need to consider two cases:

Case 1: $|\mu(c)|<q_{c}$

Let $x_{s}^{\prime}=c \quad$ and $\quad x_{c}^{\prime}=\mu(c) \cup\{s\}$. Then $h\left(x_{c}^{\prime}, x_{s}^{\prime}, x_{-\{c, s\}}\right)(s)=c \quad$ and $h\left(x_{c}^{\prime}, x_{s}^{\prime}, x_{-\{c, s\}}\right)(c)=\mu(c) \cup\{s\}$. But $x \in S N(\Lambda, R)$ which implies either

or

$$
\mu(s)=h(x)(s) P_{s} h\left(x_{c}^{\prime}, x_{s}^{\prime}, x_{-\{c, s\}}\right)(s)=c
$$

$$
\mu(c)=h(x)(c) P_{c} h\left(x_{c}^{\prime}, x_{s}^{\prime}, x_{-\{c, s\}}\right)(c)=\mu(c) \cup\{s\}
$$

Thus the college-student pair $(c, s)$ does not block $\mu$ under $R$.

Case 2: $|\mu(c)|=q_{c}$

Let $x_{s}^{\prime}=c$ and $x_{c}^{\prime}=\left(\mu(c) \backslash\left\{s^{\prime}\right\}\right) \cup\{s\}$ for some student $s^{\prime} \in \mu(c)$. Then $h\left(x_{c}^{\prime}, x_{s}^{\prime}, x_{-\{c, s\}}\right)(s)=c$ and $h\left(x_{c}^{\prime}, x_{s}^{\prime}, x_{-\{c, s\}}\right)(c)=\left(\mu(c) \backslash\left\{s^{\prime}\right\}\right) \cup\{s\}$. But $x \in S N(\Lambda, R)$ which implies either

or

$$
\mu(s)=h(x)(s) P_{s} h\left(x_{c}^{\prime}, x_{s}^{\prime}, x_{-\{c, s\}}\right)(s)=c
$$

$$
\mu(c)=h(x)(c) P_{c} h\left(x_{c}^{\prime}, x_{s^{\prime}}^{\prime}, x_{-\{c, s\}}\right)(c)=\left(\mu(c) \backslash\left\{s^{\prime}\right\}\right) \cup\{s\}
$$

But $s^{\prime} \in \mu(c)$ is arbitrary, therefore either

$$
\mu(s) P_{s} c
$$

or

$$
\forall s^{\prime} \in \mu(c) \quad \mu(x) P_{c}\left(\mu(c) \backslash\left\{s^{\prime}\right\}\right) \cup\{s\}
$$

Thus the college-student pair $(c, s)$ does not block $\mu$ under $R$.

Hence $\mu \in \mathscr{S}(R)$. 
Claim 2: $\mathscr{S}(R) \subseteq h(S N(\Lambda, R))$.

Let $\mu \in \mathscr{M}$ be such that $\mu \notin h(S N(\Lambda, R))$. Suppose $\mu \in \mathscr{S}(R)$. Let $x \in X$ be such that $x_{i}=\mu(i)$ for all $i \in C \cup S$. We have $x \notin S N(\Lambda, R)$. Therefore there is a $G^{\prime} \subseteq C \cup S$ and $x_{G^{\prime}}^{\prime} \in X_{G^{\prime}}$ such that $h\left(x_{G^{\prime}}^{\prime}, x_{-G^{\prime}}\right)(i) R_{i} h(x)(i)=\mu(i)$ for all $i \in G^{\prime}$, $h\left(x_{G^{\prime}}^{\prime}, x_{-G^{\prime}}\right)(j) P\left(j(x)(j)=\mu(j)\right.$ for some $j \in G^{\prime}$, and $h(x)(k) R_{k} h\left(x_{G^{\prime}}^{\prime}, x_{-G^{\prime}}\right)(k)$ for all $k \in(C \cup S) \backslash G^{\prime}$. Let

$$
G^{\prime \prime}=\left\{i \in G^{\prime}: h\left(x_{G^{\prime}}^{\prime}, x_{-G^{\prime}}\right)(i) I_{i} h(x)(i)=\mu(i)\right\}
$$

or equivalently

$$
G^{\prime \prime}=\left\{i \in G^{\prime}: h\left(x_{G^{\prime}}^{\prime}, x_{-G^{\prime}}\right)(i)=\mu(i)\right\}
$$

Then the coalition $G=G^{\prime} \backslash G^{\prime \prime}$ is such that $h\left(x_{G}^{\prime}, x_{-G}\right)(i) P_{i} h(x)(i)$ for all $i \in G$.

For all $s \in G$ and for all $y_{G} \in X_{G}$ we have

$$
h\left(y_{G}, x_{-G}\right)(s) \subseteq G \cup \mu(s)
$$

But $h\left(x_{G}^{\prime}, x_{G}\right)(s) P_{s} h(x)(s)=\mu(s) R_{s} \varnothing$, therefore $h\left(x_{G}^{\prime}, x_{-G}\right)(s) \in G$. For all $c \in G$, for all $y_{G} \in X_{G}$ we have

$$
h\left(y_{G}, x_{-G}\right)(c) \subseteq \mu(c) \cup G
$$

Let $\mu^{\prime}=h\left(x_{G}^{\prime}, x_{-G}\right)$. Then

1. $\forall s \in S \cap G \mu^{\prime}(s) \in G$,

2. $\forall s \in S \cap G \mu^{\prime}(s) P_{s} \mu(s)$,

3. $\forall c \in C \cap G \quad i \in \mu^{\prime}(c) \Rightarrow i \in G \cup \mu(c)$,

4. $\forall c \in C \cap G \mu^{\prime}(c) P_{c} \mu(c)$.

Therefore $\mu$ is not group stable contradicting $\mu$ is stable. Hence $\mu \notin \mathscr{S}(R)$.

Corollary 3 Let $\varphi \subseteq \mathscr{S}$ be strong Nash implementable. Then $\varphi=\mathscr{P}$.

Proof: Follows from Corollary 1 and Proposition 3.

What about the Pareto efficient and individually rational rule itself. The following game form due to Maskin [9] implements the Pareto and individually rational rule in strong Nash equilibria: $\Omega=(X, h)$ where $X_{i}=\mathscr{A}$ for all $i \in C \cup S$, and $h: X \rightarrow \mathscr{M}$ is such that

$$
\forall x \in X, \forall i \in C \cup S \quad h(x)(i)= \begin{cases}x_{i}(i) & \text { if } x_{i}=x_{j} \text { for all } j \in C \cup S \\ \varnothing & \text { otherwise }\end{cases}
$$

The reader is referred to Maskin [9] for a proof.

We finally consider the strong Nash implementation of the individually rational rule. The next proposition due to Kara and Sönmez [7] provides an indirect answer to this question. We state and prove this proposition in the general framework.

Lemma 3 A rule $\varphi: \mathscr{R} \rightarrow A$ is strong Nash implementable only if for all $R \in \mathscr{R}$ and for all $a, a^{\prime} \in \varphi(R)$, if $a P_{i} a^{\prime}$ for some $i \in N$, then $a^{\prime} P_{j} a$ for some $j \in N$. 
Proof: Let $\varphi$ be implementable in strong Nash equilibria and $\Gamma=(X, h)$ be the game form implementing $\varphi$ in strong Nash equilibria. Suppose there exists $R \in \mathscr{R}$ and $a, a^{\prime} \in \varphi(R)$ such that

$$
\forall i \in N \quad a R_{i} a^{\prime} \text { and } \exists j \in N \quad a P_{j} a^{\prime}
$$

Since $a, a^{\prime} \in \varphi(R)$ and $\varphi$ is strong Nash implementable there exists strategy profiles $x, x^{\prime} \in S N(\Gamma, R)$ such that $h(x)=a$ and $h\left(x^{\prime}\right)=a^{\prime}$. Consider the grand coalition $N$. We have $h(x)(i) R_{i} h\left(x^{\prime}\right)(i)$ for all $i \in N$, and $h(x)(j) P_{j} h\left(x^{\prime}\right)(j)$ for some $j \in N$ contradicting $x^{\prime} \in S N(\Gamma, R)$, completing the proof.

Proposition 4 The individually rational rule is not strong Nash implementable.

Proof: Follows from Lemma 3.

\section{Appendix A}

In Appendix $A$ we prove that the preference relation $\tilde{R}_{c}$ that is constructed in the proof of Theorem 1 is responsive for all $c \in C$.

Let us first consider the case $|\hat{S}(\mu, c)|=0$. Then $\widetilde{R}_{c}=R_{c}^{0}=R_{c}$ and we are done.

Let $|\hat{S}(\mu, c)|>0$. It suffices to show that $R_{c}^{1}$ is responsive. (The same arguments can be repeatedly used to show that $\widetilde{R}_{c}$ is responsive.)

Recall that

$$
Y_{1}(\mu, c)=\left\{G \in \Sigma_{c}^{q}: \hat{s}_{1} \notin G,|G|<q_{c}\right\}
$$

(We simply denote $Y_{1}(\mu, c)$ by $Y_{1}$.)

Let $R_{c}^{1} \in \mathscr{R}$ be such that (see Figure 3 )

1. For all $G, G^{\prime} \in \Sigma_{c}^{q \backslash} \backslash Y_{1}$

$$
G P_{c}^{1} G^{\prime} \Leftrightarrow G P_{c} G^{\prime}
$$

2. For all $G \in Y_{1}$,

$$
G P_{c}^{1} G \cup\left\{\hat{s}_{1}\right\} \text { and } \nexists \exists G^{\prime} \in \Sigma_{c}^{q} \backslash\{G\} \quad \text { such that } G P_{c}^{1} G^{\prime} P_{c}^{1} G \cup\left\{\hat{s}_{1}\right\}
$$

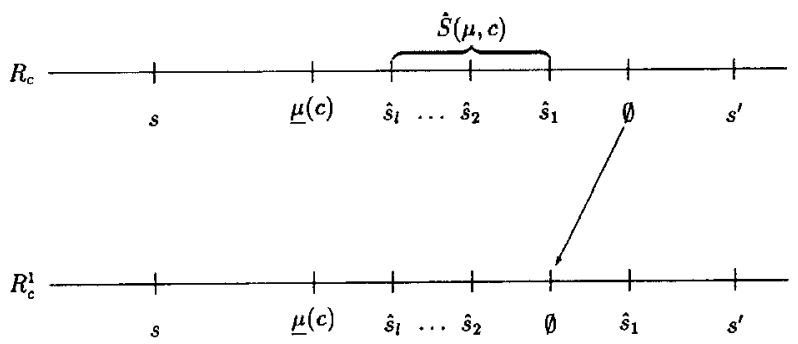

Figure 3. 
Note that (1) and (2) above and the responsiveness of $R_{c}$ implies that

$$
\forall s, s^{\prime} \in S \quad s P_{c}^{1} s^{\prime} \Leftrightarrow s P_{c} s^{\prime}
$$

Let $G \in \Sigma_{c}^{q}$ be such that $|G|<q_{c}$. We first show that for all $s, s^{\prime} \in S \backslash G$, $G \cup\{s\} P_{c}^{1} G \cup\left\{s^{\prime}\right\}$ if and only if $s P_{c}^{1} s^{\prime}$. We need to consider four cases.

(1a) $G \cup\{s\}, G \cup\left\{s^{\prime}\right\} \in \Sigma_{c}^{q} \backslash Y_{1}$.

$$
G \cup\{s\} P_{c}^{1} G \cup\left\{s^{\prime}\right\} \Leftrightarrow G \cup\{s\} P_{c} G \cup\left\{s^{\prime}\right\}
$$

(By the construction of $R_{c}^{1}$ )

$$
\Leftrightarrow s P_{c} s^{\prime}
$$

(By the responsiveness of $R_{c}$ )

$$
\Leftrightarrow s P_{c}^{1} s^{\prime}
$$

(By the construction of $R_{c}^{1}$ )

(1b) $G \cup\{s\}, G \cup\left\{s^{\prime}\right\} \in Y_{1}$

$$
\begin{aligned}
& G \cup\{s\} P_{c}^{1} G \cup\left\{s^{\prime}\right\} \Leftrightarrow G \cup\{s\} P_{c}^{1} G \cup\left\{s, \hat{s}_{1}\right\} P_{c}^{1} G \cup\left\{s^{q}\right\} P_{c}^{1} G \underbrace{\cup\left\{s_{1}^{\prime}, \hat{s}_{1}\right\}}_{\in \Sigma_{c}^{q} \backslash Y_{1}} \\
& \text { (By the construction of } R_{c}^{1} \text { ) } \\
& \Leftrightarrow s P_{c}^{1} s^{\prime}
\end{aligned}
$$

(By case 1a)

(1c) $G \cup\{s\} \in Y_{1}, G \cup\left\{s^{\prime}\right\} \in \Sigma_{c}^{q} \backslash Y_{1}$

Since $G \cup\{s\} \in Y_{1}$ we have $|G \cup\{s\}|<q_{c}$ and $\hat{s}_{1} \notin G$. This implies that $\left|G \cup\left\{s^{\prime}\right\}\right|<q_{c}$. Since any element in $\Sigma_{c}^{q} \backslash Y_{1}$ either contains $\hat{s}_{1}$ or has cardinality $q_{c}$ we have $s^{\prime}=\hat{s}_{1}$. Hence

$$
\begin{aligned}
& G \cup\{s\} P_{c}^{1} G \cup\left\{s^{\prime}\right\}=G \cup\left\{\hat{s}_{1}\right\} \Leftrightarrow G \cup\{s\} P_{c}^{1} G \cup\{s, \hat{s}_{\left.\epsilon \Sigma_{c}^{q}\right\} Y_{1}}^{\cup} P_{c}^{1} \underbrace{G \cup\left\{\hat{s}_{1}\right\}}_{E \Sigma_{c}^{q} \backslash Y_{1}} \\
& \text { (By the construction of } R_{c}^{1} \text { ) } \\
& \Leftrightarrow G \cup\left\{s, \hat{s}_{1}\right\} P_{c} G \cup\left\{\hat{s}_{1}\right\} \\
& \Leftrightarrow s P_{c} \varnothing \\
& \Leftrightarrow s P_{c} \hat{s}_{1} \\
& \Leftrightarrow s P_{c}^{1} \hat{s}_{1}=s^{\prime}
\end{aligned}
$$

(By the construction of $R_{c}^{1}$ ) 
(1d) $G \cup\{s\} \in \Sigma_{c}^{q} \backslash Y_{1}, G \cup\left\{s^{\prime}\right\} \in Y_{1}$

Since $G \cup\left\{s^{\prime}\right\} \in Y_{1}$ we have $\left|G \cup\left\{s^{\prime}\right\}\right|<q_{c}$ and $\hat{s}_{1} \notin G$. This implies that $|G \cup\{s\}|<q_{c}$. Since any element in $\Sigma_{c}^{q} \backslash Y_{1}$ either contains $\hat{s}_{1}$ or has cardinality $q_{c}$ we have $s=\hat{s}_{1}$. Hence

$$
\begin{aligned}
G \cup\{s\}=G \cup\left\{\hat{s}_{1}\right\} P_{c}^{1} G \cup\left\{s^{\prime}\right\} \Leftrightarrow \underbrace{\left.G \hat{s}_{1}\right\} P_{c}^{1} G \cup\left\{s^{\prime}\right\} P_{c}^{1} G \cup\left\{s^{\prime}, \hat{s}_{1}\right\}}_{\substack{\epsilon \Sigma_{c}^{q} \backslash Y_{1} \\
\left(\text { By the construction of } R_{c}^{1}\right)}} \\
\Leftrightarrow \sum_{c}^{q} \backslash Y_{1} \\
\Leftrightarrow \cup\left\{\hat{s}_{1}\right\} P_{c} G \cup\left\{s^{\prime}, \hat{s}_{1}\right\} \\
\quad\left(\text { By the construction of } R_{c}^{1}\right) \\
\Leftrightarrow \varnothing P_{c} s^{\prime} \\
\quad\left(\text { By the responsiveness of } R_{c}\right) \\
\Leftrightarrow \hat{s}_{1} P_{c} s^{\prime} \\
\quad\left(\text { By the definition of } \hat{s}_{1}\right) \\
\Leftrightarrow s=\hat{s}_{1} P_{c}^{1} s^{\prime}
\end{aligned}
$$

(By the construction of $R_{c}^{1}$ )

Next we show that for any $s \in S \backslash G$ we have $G \cup\{s\} P_{c}^{1} G$ if and only if $s P_{c}^{1} \varnothing$. We need to consider three cases.

\section{(2a) $G \in \Sigma_{c}^{q} \backslash Y_{1}$}

Since $|G|<q_{c}, G \in \Sigma_{c}^{q} \backslash Y_{1}$ implies that $\hat{s}_{1} \in G$. Thus $s \neq \hat{s}_{1}$ and $G \cup\{s\} \in$ $\Sigma_{c}^{q} \backslash Y_{1}$. Hence

$$
G \cup\{s\} P_{c}^{1} G \Leftrightarrow G \cup\{s\} P_{c} G
$$

(By the construction of $R_{c}^{1}$ )

$$
\Leftrightarrow s P_{c} \varnothing
$$

(By the responsiveness of $R_{c}$ )

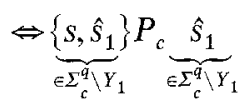

(By the responsiveness of $R_{c}$ )

$$
\Leftrightarrow\left\{S, \hat{s}_{1}\right\} P_{c}^{1} \hat{s}_{1}
$$

(By the construction of $R_{c}^{1}$ )

$$
\Leftrightarrow s P_{c}^{1}\left\{s, \hat{s}_{1}\right\} P_{c}^{1} \varnothing P_{c}^{1} \hat{s}_{1}
$$

(By the construction of $R_{c}^{1}$ )

$\Leftrightarrow s P_{c}^{1} \varnothing$ 
(2b) $G, G \cup\{s\} \in Y_{1}$

$$
G \cup\{s\} P_{c}^{1} G \Leftrightarrow G \cup\{s\} P_{c}^{1} \underbrace{G \cup\left\{s, \hat{s}_{1}\right\}}_{\in \Sigma_{c}^{q} \backslash Y_{1}} P_{c}^{1} G P_{c}^{1} \frac{G \cup\left\{\hat{s}_{1}\right\}}{\in \Sigma_{c}^{q^{q}} \backslash Y_{1}}
$$

(By the construction of $R_{c}^{1}$ )

$$
\Leftrightarrow s P_{c}^{1} \varnothing
$$

(By case 2a)

(2c) $G \in Y_{1}, G \cup\{s\} \in \Sigma_{c}^{q} \backslash Y_{1}$

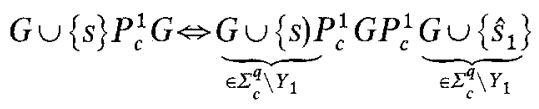

(By the construction of $R_{c}^{1}$ )

$$
\Leftrightarrow s P_{c}^{1} \hat{s}_{1}
$$

(By the case 1a)

$$
\Leftrightarrow s P_{c}^{1} \varnothing
$$

(By the construction of $R_{c}^{1}$ )

Hence $R_{c}^{1}$ is responsive.

\section{Appendix B}

In Appendix B we prove that the preference relation $\widetilde{R}$ that is constructed in the proof of Theorem 1 is such that $R \in M T(\tilde{R}, \mu)$.

Let $R \in \mathscr{R}$ and $\mu \in \mathscr{S}(R)$. Let $\widetilde{R}$ be defined as in the proof of Theorem 1. Let $s \in S$. We have $L\left(\mu, \tilde{R}_{s}\right)=L\left(\mu, R_{s}\right)$ by construction.

Let $c \in C$. If $\tilde{R}_{c}=R_{c}$ then $L\left(\mu, \tilde{R}_{c}\right)=L\left(\mu, R_{c}\right)$. Suppose $\tilde{R}_{c} \neq R_{c}$, then $|\mu(c)|=q_{c}$ and $\mu(c) \in \Sigma_{c}^{q} \backslash Y_{1}(\mu, c)$. Let $\mu^{\prime} \in L\left(\mu, R_{c}^{1}\right)$ thus $\mu(c) R_{c}^{1} \mu^{\prime}(c)$. If $\mu^{\prime}(c) \in \Sigma_{c}^{q} \backslash Y_{1}(\mu, c)$, then $\mu(c) R_{c} \mu^{\prime}(c)$ by construction. If $\mu^{\prime}(c) \in Y_{1}(\mu, c)$ then $G=\left(\mu^{\prime}(c) \cup\left\{\hat{s}_{1}\right\}\right) \in$ $\Sigma_{c} \backslash Y_{1}(\mu, c)$ and $\mu^{\prime}(c) P_{c}^{1} G$ by construction. Therefore $\mu(c) R_{c}^{1} \mu^{\prime}(c) P_{c}^{1} G$. But we have $\mu(c), G \in \Sigma_{c}^{q} \backslash Y_{1}(\mu, c)$ therefore $\mu(c) P_{c} G$ by construction. We also have $G P_{c} \mu^{\prime}(c)$ which implies $\mu(c) P_{c} \mu^{\prime}(c)$. In either case we have $\mu(c) R_{c} \mu^{\prime}(c)$ therefore $L\left(\mu, R_{c}^{1}\right) \subseteq L\left(\mu, R_{c}\right)$. The same arguments can be repeatedly used to show $L\left(\mu, \widetilde{R}_{c}\right) \subseteq L\left(\mu, R_{c}\right)$. Hence $R \in M T(\widetilde{R}, \mu)$.

\section{References}

1. Alcalde, J., Barberà, S.: Top dominance and the possibility of strategy-proof stable solutions to matching problems. Econ. Theory 4, 417-435 (1994)

2. Danilov, V.: Implementation via Nash equilibria. Econometrica 60, 43-56 (1992)

3. Dutta, B., Sen, A.: A necessary and sufficient condition of two-person Nash implementation. Rev. Econ. Stud. 58, 121-128 (1991)

4. Gale, D., Shapley, L.: College admissions and the stability of marriage. Amer. Math. Monthly 69, 9-15 (1962) 
5. Gale, D., Sotomayor, M.: Some remarks on the stable matching problem. Discrete Appl. Math. 11, 223-232 (1985)

6. Kara, T., Sönmez, T.: Nash implementation of matching rules. University of Rochester, mimeo 1993. Journal of Economic Theory, forthcoming

7. Kara, T., Sönmez, T.: Strong implementation in matching games. University of Rochester, mimeo 1994

8. Maskin, E.: Nash equilibrium and welfare optimality. MIT, mimeo 1977

9. Maskin, E.: Implementation and strong Nash equilibrium. In: J.-J. Laffont (ed.) Aggregation and revelation of preferences. Amsterdam New York Oxford: North-Holland 1979

10. Maskin, E.: The theory of implementation in Nash equilibrium: A survey. In: L. Hurwicz, D. Schmeidler, H. Sonnenschein (eds.) Social goals and social organization: volume in memory of Elisha Pazner. London New York: Cambridge University Press 1985

11. Moore, J.: Implementation in environments with complete information. In: J.-J. Laffont (ed.) Advances in economic theory. London New York: Cambridge University Press 1992

12. Moore, J., Repullo, R.: Nash implementation: A full characterization. Econometrica 58, 1083-1099 (1990)

13. Palfrey, T. R., Srivastava, S.: Nash implementation using undominated strategies. Econometrica 59, 479-501 (1991)

14. Roth, A. E.: The economics of matching: stability and incentives. Math. Oper. Res. 7, 617-628 (1982)

15. Roth, A. E.: The evolution of the labor market for medical interns and residents: a case study in game theory. J. Polit. Econ. 92, 991-1016 (1984)

16. Roth, A. E.: The college admissions problem is not equivalent to the marriage problem. J. Econ. Theory 36, 277-288 (1985)

17. Roth, A. E.: Common and conflicting interests in two-sided matching markets. Eur. Econ. Rev. 27, 75-96 (1985)

18. Roth, A. E.: On the allocation of residents to rural hospitals: a general property of two-sided matching markets. Econometrica 54, 425-427 (1986)

19. Roth, A. E., Sotomayor, M.: The college admissions problem revisited. Econometrica 57, 559-570 (1989)

20. Roth, A. E., Sotomayor, M.: Two-sided matching: a study in game theoretic modeling and analysis. London New York: Cambridge University Press 1990

21. Saijo, T:: Strategy space reduction in Maskin's theorem: sufficient conditions for Nash implementation. Econometrica 56, 693-700 (1988)

22. Sen, A.: Approximate implementation of non-dictatorial social choice functions. Princeton University, mimeo 1987

23. Sjöström, T.: On the necessary and sufficient conditions for Nash implementation. Soc. Choice Welfare 8, 333-340 (1991)

24. Sönmez, T.: Strategy-proofness in many-to-one matching problems. University of Rochester, mimeo 1994. Economic Design, forthcoming

25. Sönmez, T.: Strategy-proofness and singleton cores in generalized matching problems. University of Rochester, mimeo 1995

26. Thomson, W.: Minimal monotonic extensions. University of Rochester, mimeo 1992

27. Thomson, W.: Concepts of implementation. University of Rochester, mimeo 1993

28. Williams, S.: Realization and Nash implementation: two aspects of mechanism design. Econometrica 54, 139-151 (1986)

29. Yamato, T.: On Nash implementation of social choice correspondences. Games Econ. Behav. 4, 484-492 (1992) 